\title{
ÍNDICE DE SERVIÇO ADEQUADO PARA AS EMPRESAS DE NAVEGAÇÃO FLUVIAL DE PASSAGEIROS DO AMAZONAS
}

\author{
José Teixeira de Araújo Neto Santos \\ Instituto de Pesquisa em Transportes - INTRA \\ teixeira.santos@intra.org.br \\ Poliana Cardoso \\ Instituto de Pesquisa em Transportes - INTRA \\ polianacard@yahoo.com.br \\ Nelson Kuwahara \\ Universidade Federal do Amazonas - UFAM \\ kuwaharanelson@yahoo.com \\ Waltair Vieira Machado \\ Universidade Federal do Amazonas - UFAM \\ waltair_machado@yahoo.com.br
}

\begin{abstract}
Resumo
O transporte fluvial misto da Região Amazônica apresenta ineficiências, principalmente, no que diz respeito às condições das embarcações mistas que operam na Região. No geral, estas embarcações não atendem as legislações do setor de transporte aquaviário, o que resulta em riscos aos usuários. Neste trabalho, o objetivo é propor uma metodologia para determinar o desempenho global das empresas de navegação mista, em função dos critérios de serviços adequados descritos na resolução 912 da Agência Nacional de Transportes Aquáticos - ANTAQ. A ocorrência de mais de uma variável na definição do desempenho das empresas de navegação torna o problema multicritério, ou seja, um conjunto de empresas (alternativas) é avaliado por múltiplos critérios conflitantes de serviços adequados, tais como: segurança, atendimento, higiene, conforto, modicidade e alimentação. A abordagem proposta será demostrada por meio de um estudo de caso real realizado em um conjunto de empresas de transporte de passageiro e misto na Amazônia. O modelo proposto permite definir o desempenho das empresas de Navegação.
\end{abstract}

Palavras-Chaves: Desempenho; Transporte fluvial; Amazônia.

\begin{abstract}
The inland waterways of the Amazon region presents mixed inefficiencies, especially with regard to the conditions of mixed vessels operating in the region. Overall, these vessels do not meet the laws of the waterway transport sector, resulting in risks to users. In this work, the goal is to propose a methodology to determine the overall performance of shipping companies mixed, according to the criteria of appropriate services described in Resolution 912 of the Agência Nacional de Transportes Aquáticos - ANTAQ. The occurrence of more than one variable in defining the performance of shipping companies makes the multicriteria problem, ie, a set of companies (alternatives) is rated by multiple conflicting criteria of appropriate services, such as security, care, hygiene, comfort, affordability and power. The proposed approach will demostrada through a real case study conducted in a number of transport companies and passenger mix on Amazon. The proposed model allows to define the performance of companies Navigation.
\end{abstract}

Keywords: Performance; Inland waterways; Amazon. 


\section{INTRODUÇÃO}

$\mathrm{Na}$ Amazônia o transporte de passageiros e mercadorias é realizado, principalmente, por embarcações mistas (mercadorias e passageiros), construídas em estaleiros e/ou carreiras situadas em alguns municípios do Amazonas.

As embarcações mistas utilizadas no transporte fluvial da Amazônia são de concepção artesanal, a maioria é de madeira e não são desenvolvidas segundo as técnicas e recomendações da engenharia. A consequência deste cenário são os acidentes, provocados pela dificuldade de navegabilidade e operacionalidade das embarcações [7][8].

As embarcações que realizam este transporte representam altos riscos para os usuários, em virtude da falta de qualidade na manutenção, do desconforto das acomodações, da superlotação, do carregamento desordenado e da baixa qualidade dos alimentos servidos [8].

Em função da qualidade e da confiabilidade do serviço de transporte, os municípios interioranos da Amazônia não conseguem se inserir na economia de mercado [9]. Pois, as condições das embarcações influenciam na competitividade do setor produtivo regional e contribuem para que os municípios da Amazônia não sejam atrativos ao capital produtivo.

Os indicadores de serviço adequado são uma ferramenta propícia para auxiliar as tomadas de decisão, influenciando no planejamento das políticas públicas, na regulamentação, qualificação e especialização do setor, além de fornecer alinhamento conceitual aos usuários [6].

Desta forma, o objetivo principal deste trabalho é apresentar uma metodologia para determinar o desempenho global das empresas de navegação fluvial mista, adaptada às características regionais, e assim, auxiliar na estruturação do sistema de transporte fluvial da Região Amazônica.

Apesar da existência de modelos que tratam desta problemática, estes fornecem resultados pouco satisfatórios, uma vez que, há muitas situações que não tem dados objetivos e, além de deseja-se observar o comportamento em mais de um critério. Neste contexto, o artigo faz uso do método multicritérios PROMETHEE (Preference Ranking Organization Method for Enrichment and Evaluation) para determinar o desempenho global das empresas de navegação, analisando múltiplos critérios de serviço adequado. O método PROMETHEE, consiste em construir e explorar uma relação de sobreclassificação de valores [3].

O trabalho está divido em seis seções, sendo a Seção 1 a presente introdução. $\mathrm{Na}$ Seção 2 é apresentada a revisão da literatura. A Seção 3 apresenta a metodologia de pesquisa e o método PROMETHEE, a Seção 4 apresenta os dados do estudo de caso abordado e a Seção 5 apresenta os resultados e a análise de sensibilidade. Por fim, a Seção 6 apresenta as considerações finais.

\section{REVISÃO DA LITERATURA}

[11] desenvolveu um conjunto de indicadores de desempenho que possibilitam avaliar os serviços de transporte longitudinal misto. No trabalho são construídos índices de operacionalidade e qualidade para apontar a frequência relativa e destacar as empresas de navegação com melhores práticas. O índice de operacionalidade é obtido a partir das características operacionais das empresas transporte. Já o índice de qualidade, depende de informações da qualidade dos serviços prestados, que são subjetivas.

[6] desenvolveu um indicador global, utilizando Análise Envoltória de Dados (Data Envelopment Analysis - DEA), para avaliar o transporte aquaviário de passageiros da Região Amazônica. No modelo, os autores definiram a viagem como insumo (inputs); a segurança, o atendimento, a higiene, o conforto, a modicidade e a alimentação como produtos (outputs); e por fim as embarcações como Unidades Tomadoras de Decisão (Decison Making Units $D M U s)$. A pesquisa fornece a eficiência das embarcações e os benchmarks para as menos eficientes.

Já [7] realizou uma análise ergonômica nas embarcações da região com o objetivo de 
propor melhorias para as mesmas. A pesquisa baseou-se nos procedimentos de delimitação e categorização dos problemas ergonômicos, onde foram levantadas deficiências e falhas específicas, para chegar a uma proposta ergonômica que atenda de modo eficiente as necessidades do sistema.

[8] apresenta duas metodologias de gestão da qualidade que podem auxiliar os gestores na tomada de decisões voltadas à melhoria da qualidade do serviço. Neste caso, o autor avaliou o transporte de passageiros na Amazônia Ocidental com foco nos serviços oferecidos aos usuários. Para o autor, a baixa qualidade oferecida no transporte aquaviário de passageiros é ocasionada pela falta de compromisso das autoridades públicas em regulamentar o transporte na região.

Com base na revisão da literatura conclui-se que, a problemática apresenta as seguintes características: o número de critérios é maior que dois e conflitantes; a solução depende de um conjunto de pessoas, alguns critérios são quantificáveis ao passo que outros só o são por meio de julgamento subjetivo e por fim, os problemas não estão claramente definidos e estruturados.

De um modo geral, as considerações finais dos trabalhos indicam que as embarcações apresentam riscos para os usuários, ausência de qualidade na manutenção, desconforto das acomodações, superlotação, carregamento desordenado e baixa qualidade dos alimentos servidos.

De fato, a variedade e complexidade do problema faz das empresas de navegação fluvial mista um campo promissor para utilização e desenvolvimento de indicadores.

\section{METODOLOGIA}

Neste artigo propõe-se a construção de um indicador de desempenho global para avaliar o serviço adequado de um conjunto de empresas de navegação. Abreviadamente, o indicador foi designado de "Índice de Serviço Adequado - ISA". O ISA é uma pontuação global de desempenho para cada empresa de navegação que realiza o transporte de passageiro ou misto, a partir dos requisitos: regularidade, eficiência, segurança, atendimento ao interesse público, generalidade, pontualidade, conforto, continuidade, atualidade, cortesia na prestação dos serviços, modicidade nas tarifas e fretes e preservação do meio ambiente a ambiental, econômica, logística e desempenho de segurança de forma integrada.

O objetivo do ISA é fornecer um índice para avaliar o desempenho global de um conjunto de empresas de navegação de acordo com um conjunto de índices de serviço adequado.

Nos problemas de Apoio Multicritério à Decisão - AMD o primeiro passo é definir uma lista de indicadores (ou critérios) de avaliação com base no objetivo estabelecido pelos tomadores de decisão. No presente caso, os critérios selecionados são descritos na resolução 912 da Agência Nacional de Transportes Aquáticos - ANTAQ, pois torna possível avaliar as empresas de navegação de acordo com os requisitos específicos de serviço adequado.

O Segundo passo é avaliar o impacto dos critérios nas empresas de navegação, e assim, podemos agrega-los em um indicador global que representa o desempenho das empresas de navegação no conjunto de indicadores de serviço adequado. A Figura 1 ilustra a estrutura ISA da metodologia apresentada. 


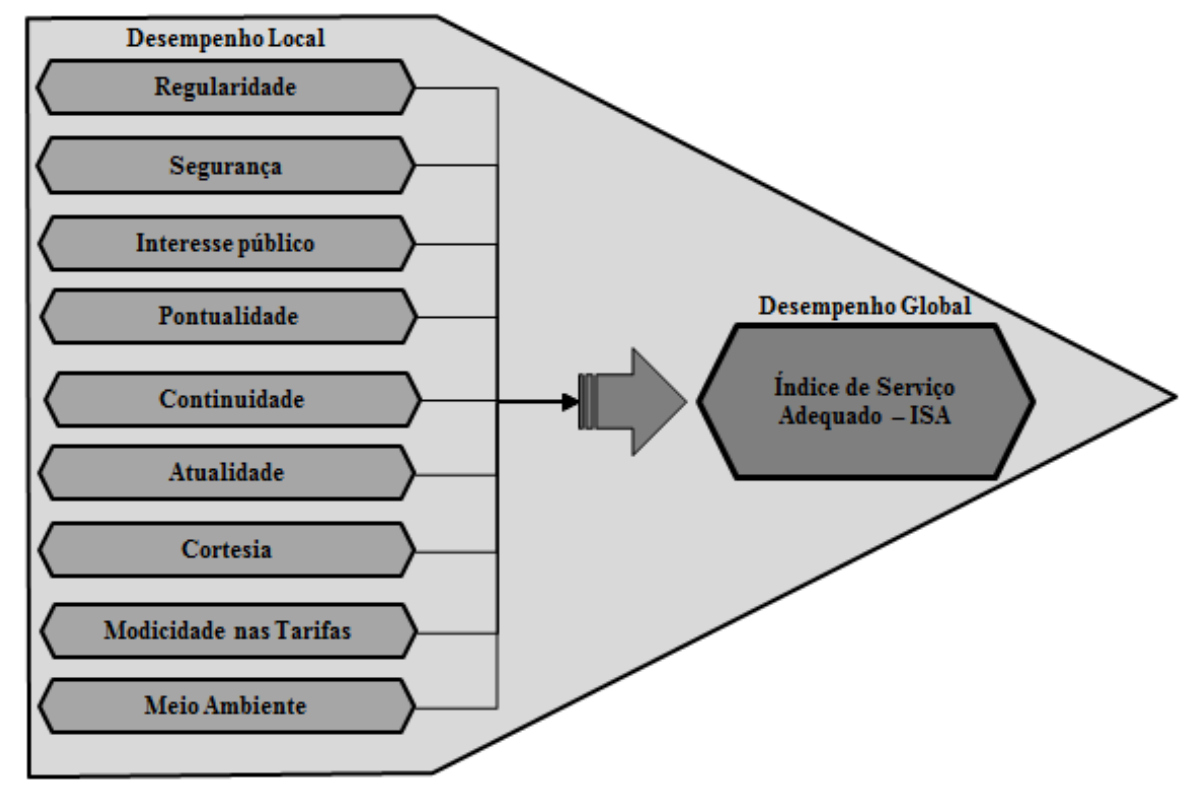

Figura 1: Estrutura da metodologia ISA.

Fonte: [12] adaptado.

Neste trabalho, a etapa de agregação é realizada por meio de um método multicritérios, especificamente, o PROMETHEE, apresentado principalmente por [2]. O método de sobreclassificação (outranking) PROMETHEE trata de matrizes semelhantes, onde tem se um conjunto A finito e enumerável de $\mathrm{n}$ alternativas e um conjunto de $k$ de $m$ critérios de avaliação.

O PROMETHEE se baseia na comparação par-a-par e envolve a definição de vários parâmetros que permitem ajustar a abordagem e modelar o problema o mais corretamente possível [10]. No caso do problema apresentado, este método permite avaliações quantitativas e qualitativas e uma vantagem não negligenciável, no caso de uso da escala linguística.

O PROMETHEE exige informações para cada critério, assim uma função específica de preferência deve ser definida e usada para calcular o grau de preferência associado à melhor alternativa em caso de comparações par-a-par. Há seis funções de preferência descritas por [4]. O índice de preferência multicritério é computado como segue:

$$
\left\{\begin{array}{l}
\pi(a, b)=\sum_{j=1}^{k} w_{j} P_{j}(a, b) \\
\pi(b, a)=\sum_{j=1}^{k} w_{j} P_{j}(b, a)
\end{array}\right.
$$

Onde $w_{j}$ é o peso do critério e $P_{j}(a, b)$, é uma função de preferência que determina o grau de preferência de uma alternativa $a$ sobre uma alternativa $b$, leva em conta todos os critérios ao critério $f_{k}$. O $\pi(a, b)$ expressa com que grau $a$ é preferível a $b$ e $\pi(b, a)$ como $b$ é preferível a $a$, consequentemente, $\pi(a, b)$ e $\pi(b, a)$ são geralmente positivas, entre 0 e 1 .

As alternativas do conjunto A enfrenta $n-1$ alternativas em $\mathrm{A}$, onde são definidos os fluxos de sobreclassificação positivo $\phi+$ e negativo $\phi$-, segundo [4].

- $\phi^{+}(a)$ expressa o nível em que alternativa a sobreclassifica todas as outras, ou seja, quanto maior $\phi^{+}(a)$ melhor é a alternativa; 
$\phi^{+}(a)=\frac{1}{n-1} \sum_{b} \pi(a, b)$

- $\phi^{-}(a)$, determina o quanto a alternativa a é superada por todas as outras, neste caso, quanto menor $\phi^{-}(a)$, melhor é a alternativa.

$\phi-(a)=\frac{1}{n-1} \sum_{b} \pi(b, a)$

Há dois modelos PROMETHEE clássicos: PROMETHEE I e II. O PROMETHEE I fornece um pré-ordem parcial e é obtido por meio dos fluxos $\phi^{+}$e $\phi$. Já o PROMETHEE II é diferença entre os dois fluxos de preferência, ou seja, o fluxo de preferência líquido (4), que estabelece uma pré -ordem completa [4].

$\phi(a)=\phi^{+}(a)-\phi^{-}(a)$

Dessa forma, obtém-se a ordenação das alternativas, de acordo com os valores encontrados pelo fluxo líquido. O fluxo líquido de critério único $\phi_{j}($.$) obtido quando um único$ critério $g_{j}($. $)$ é considerado. Ele expressa como uma alternativa a sobreclassifica $\left(\phi_{j}(a)>0\right)$ ou é desclassificada $\left(\phi_{j}(a)<0\right)$ por todas as alternativas no critério $g j($.$) . O perfil de uma$ alternativa consiste no conjunto de toda a rede do critério único de fluxos: $\phi_{j}(a), j=1,2, \ldots, k$ [4].

Neste trabalho utiliza-se o método PROMETHEE II, pois a problemática é do tipo $\mathrm{P}_{. \gamma}$ onde o resultado pretendido é uma ordenação completa, conforme as preferências.

\subsection{CRITÉrios de SERVIÇO AdEQUAdo}

Esta seção apresenta a definição dos critérios de serviço adequados. A [1] define serviço adequado como sendo aquele que é realizado de maneira a satisfazer os requisitos: regularidade, eficiência, segurança, atendimento ao interesse público, generalidade, pontualidade, conforto, continuidade, atualidade, cortesia na prestação dos serviços, modicidade nas tarifas e fretes e preservação do meio ambiente.

A [1] aponta os indicadores de serviços adequados, mas não prescreve como calculálos. Estão descritas abaixo a definição de 10 critérios, indicando quais variáveis os definem [5].

- Regularidade: Manutenção da prestação do serviço segundo padrões qualitativos e quantitativos uniformes. O critério pode ser definido por meio das variáveis: embarque e desembarque fora do local; multas; apreensões; interdições; conformidades com as normas; viagens realizadas e documentação exigida podem ser consideradas como variáveis importantes para o cálculo do indicador de regularidade do modal aquaviário.

- Segurança: desenvolvimento da atividade sem pôr em risco a integridade física e emocional de quem quer que seja - usuários e não usuários. O critério pode ser definido por meio das variáveis: acidentes, crimes e da satisfação com a segurança que é importante para o cálculo do indicador de segurança que deverá ser aferido pela 
média aritmética desses indicadores.

- Atendimento ao interesse público: O critério pode ser definido por meio das variáveis: satisfação com o preço, alimentação, conforto e higiene.

- Generalidade: Universalização da oferta do serviço, de tal forma que propicie a prestação de utilidades a todos os potenciais interessados, ainda que razões materiais e de segurança possam acarretar a limitação quantitativa da oferta do serviço. O critério pode ser definido por meio das variáveis: ocorrências às restrições de passageiros com deficiência física; ocorrências às restrições de passageiros idosos; ocorrências às restrições com crianças/adolescente; ocorrências com usuários em geral.

- Pontualidade: o rigoroso cumprimento dos horários para a prestação do serviço préestabelecido. O critério pode ser definido por meio das variáveis: saídas atrasadas; saídas antes do horário; chegadas atrasadas.

- Continuidade: Ausência de interrupção, segundo a natureza da atividade desenvolvida e do interesse a ser atendido. O critério pode ser definido por meio das variáveis: viagens não iniciadas e viagens interrompidas.

- Atualidade: o cumprimento, pela operadora, da prestação com o uso de novas técnicas, equipamentos e tecnologias em geral além de um gradual melhoramento da ampliação da capacidade de atendimento para acompanhar a demanda. O critério pode ser definido por meio das variáveis: treinamento de pessoal, conservação das instalações e modernidade.

- Cortesia na prestação dos serviços: Significa respeito à dignidade humana e tratamento civilizado. O critério pode ser definido por meio das variáveis: deficiência de atendimento no embarque, durante a viagem e desembarque.

- Modicidade nas tarifas e fretes: Serviço público deve estar à disposição de todos, não apenas formalmente como materialmente, não podendo a tarifa constranger os que necessitam dele. $\mathrm{O}$ critério pode ser definido por meio das variáveis: tarifa, gratuidade e satisfação do passageiro em relação ao preço.

- Preservação do meio ambiente. O critério pode ser definido por meio das variáveis: poluição do ar, poluição sonora gerada pela embarcação; lançamentos de dejetos; vazamento de óleo pela embarcação e consumo de combustível.

\section{ESTUDO DE CASO}

O problema proposto envolve a definição de um índice de serviço adequado para um conjunto de empresas de navegação autorizadas a oferecer o serviço de transporte de passageiro e misto da Amazônia. A pesquisa considera 28 embarcações (alternativas) que operam nos eixos considerados troncais: Manaus - Belém; Manaus - Santarém; Manaus Porto Velho; e Manaus - Tabatinga.

\subsection{DADOS DOS CRITÉRIOS}

Os dados utilizados para determinar o desempenho local dos critérios nas empresas de navegação foram extraídos do trabalho de [5]. Os dados são resultados dos cálculos de indicadores parciais que levam em consideração a satisfação dos passageiros em cada requisito da definição de serviço adequado. Os desempenhos locais encontram-se na Tabela 1.

Tabela 1: Desempenho local dos critérios de serviço adequando Critérios

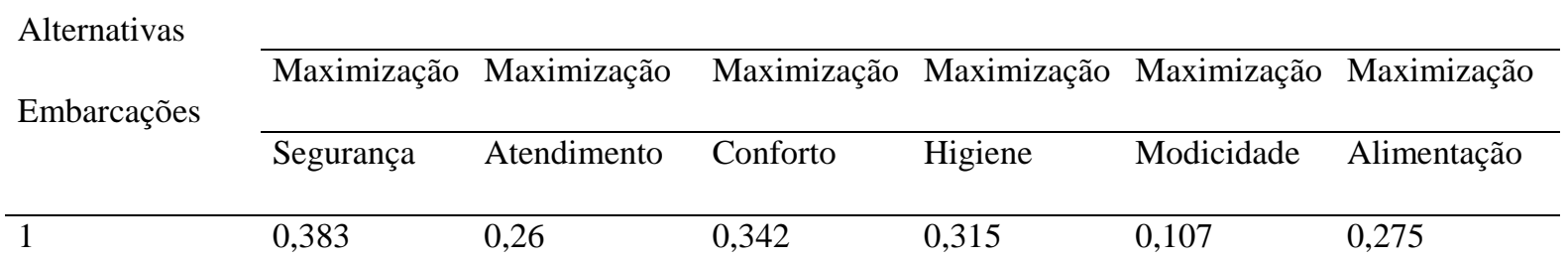




\begin{tabular}{|c|c|c|c|c|c|c|}
\hline 2 & 0,181 & 0,142 & 0,149 & 0,21 & 0,081 & 0,129 \\
\hline 3 & 0,222 & 0,156 & 0,159 & 0,173 & 0,09 & 0,201 \\
\hline 4 & 0,151 & 0,094 & 0,108 & 0,096 & 0,08 & 0,092 \\
\hline 5 & 0,167 & 0,14 & 0,14 & 0,157 & 0,1 & 0,162 \\
\hline 6 & 0,216 & 0,161 & 0,176 & 0,172 & 0,113 & 0,181 \\
\hline 7 & 0,43 & 0,259 & 0,282 & 0,392 & 0,045 & 0,316 \\
\hline 8 & 0,316 & 0,225 & 0,248 & 0,296 & 0,088 & 0,267 \\
\hline 9 & 0,126 & 0,067 & 0,082 & 0,115 & 0,047 & 0,024 \\
\hline 10 & 0,213 & 0,166 & 0,14 & 0,178 & 0,16 & 0,187 \\
\hline 11 & 0,104 & 0,147 & 0,117 & 0,16 & 0,181 & 0,13 \\
\hline 12 & 0,067 & 0,05 & 0,067 & 0,067 & 0,067 & 0 \\
\hline 13 & 0,192 & 0,171 & 0,141 & 0,204 & 0,227 & 0,111 \\
\hline 14 & 0,255 & 0,208 & 0,242 & 0,255 & 0,138 & 0,197 \\
\hline 15 & 0,211 & 0,142 & 0,158 & 0,079 & 0,222 & 0,108 \\
\hline 16 & 0,453 & 0,331 & 0,375 & 0,556 & 0,033 & 0,359 \\
\hline 17 & 0,284 & 0,225 & 0,336 & 0,248 & 0,145 & 0,172 \\
\hline 18 & 0,375 & 0,156 & 0,25 & 0 & 0,125 & 0,25 \\
\hline 19 & 0,111 & 0,15 & 0,143 & 0,213 & 0,117 & 0,129 \\
\hline 20 & 0,286 & 0,242 & 0,338 & 0,3 & 0,071 & 0,257 \\
\hline 21 & 0,405 & 0,301 & 0,38 & 0,43 & 0,103 & 0,291 \\
\hline 22 & 0,349 & 0,265 & 0,326 & 0,326 & 0,14 & 0,268 \\
\hline 23 & 0,31 & 0,208 & 0,224 & 0,309 & 0,073 & 0,224 \\
\hline 24 & 0,263 & 0,295 & 0,368 & 0,316 & 0,053 & 0,444 \\
\hline 25 & 0,222 & 0,142 & 0,111 & 0,235 & 0 & 0,444 \\
\hline 26 & 0,295 & 0,203 & 0,18 & 0,226 & 0,164 & 0,242 \\
\hline 27 & 0,538 & 0,343 & 0,399 & 0,506 & 0,07 & 0,395 \\
\hline 28 & 0,250 & 0,191 & 0,132 & 0,269 & 0,091 & 0,273 \\
\hline
\end{tabular}

\section{Fonte: [5]}

\subsection{PESOS DOS CRITÉRIOS}

Os pesos dos critérios foram determinados com base no procedimento de comparações par a par, por meio da matriz de julgamento $K=\left[k_{i j}\right]$, onde $k_{i j}$ representa a importância do critério $k_{i}$ em relação ao critério kj. A matriz de julgamento é positiva $\left(k_{i j}>\right.$ 
0 ), quadrada recíproca $n \times n$, tal que $k_{i j}=1: k_{j i}=1 / k_{i j}$ e com 1 na diagonal principal. O $k_{i j}$ assume valores de 1 a 9, conforme escala apresentada na Figura 2.

\begin{tabular}{|c|c|c|c|c|c|c|c|c|}
\hline $1 / 9$ & $1 / 7$ & $1 / 5$ & $1 / 3$ & 1 & 3 & 5 & 7 & 9 \\
\hline Extremamente & Bastante & Muito & Pouco & Igual & Pouco & Muito & Bastante & Extremamente \\
\hline
\end{tabular}

Figura 2: Escala utilizada na comparação dos critérios.

Fonte: [13] adaptado.

A importância relativa dos critérios foi determinada por três especialistas da UFAM Universidade Federal do Amazonas, por meio de entrevistas. Assim, foi possível estabelecer três ponderações diferentes para os pesos dos critérios (Figura 3). Estes pesos são:

- Peso Global: é a Prioridade Média Local - PML dos critérios obtida por meio de uma matriz de julgamento, onde $k_{i j}$ é o valor médio da importância relativa atribuída para cada critério.

- Peso Mínimo: é a PML dos critérios obtida por meio de uma matriz de julgamento, onde $k_{i j}$ é o menor valor de importância relativa atribuída para cada critério.

- Peso Máximo: é a PML dos critérios obtida por meio de uma matriz de julgamento, onde $k_{i j}$ é o maior valor de importância relativa atribuída para cada critério.

A comparação dos resultados finais obtidos em diferentes pesos é uma forma de testar a sensibilidade do modelo. Esta é uma análise pós-avaliação e é importante para fornecer uma recomendação robusta [12].

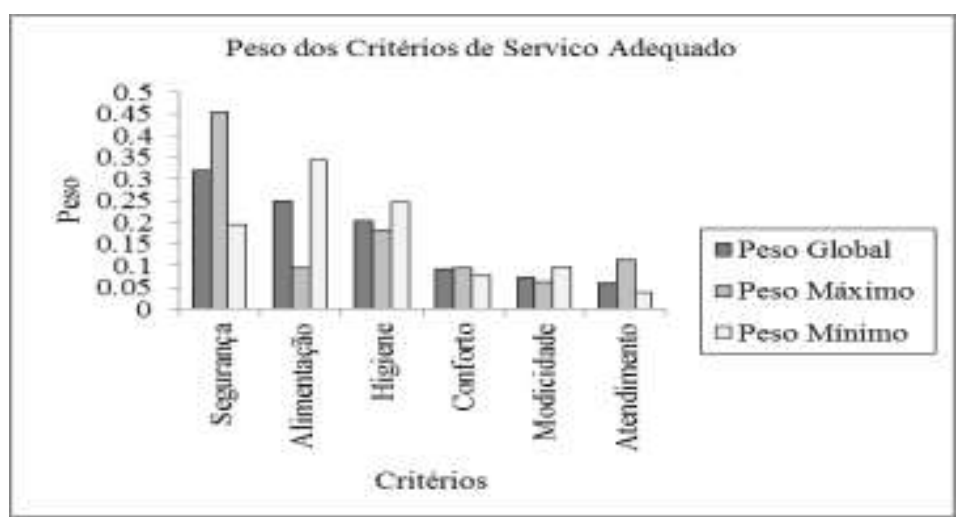

Figura 3: Pesos (global, máximo e mínimo) dos critérios.

A Figura 3 apresenta o resultado referente aos pesos global, máximo e mínimo. Observa-se com relação ao peso global, que a segurança $(31.9 \%)$ é considerada a mais importância, seguida da alimentação $(24.9 \%)$, higiene $(20.4 \%)$, conforto $(9.3 \%)$, modicidade $(7.4 \%)$ e atendimento $(6.1 \%)$. No contexto do peso máximo, a segurança $(45.4 \%)$ conseguiu a maior importância relativa, seguida da higiene $(18.1 \%)$, atendimento $(11.4 \%)$, alimentação (9.5\%), conforto $(9.3 \%)$ e modicidade $(6.2 \%)$. Já no peso mínimo, o resultado indica que a alimentação (34.5\%) tem a maior importância, seguida da higiene $(24,7 \%)$, segurança $(19,4 \%)$, Modicidade (9.7\%), conforto (8\%) e atendimento (3.8).

$\mathrm{Na}$ análise comparativa apresentada nesta seção, observa-se que a ponderação do peso global apresenta um resultado semelhante aos pesos máximo e mínimo. Desta forma conclui-se que, os três tomadores de decisão tem o mesmo ponto de vista.

No método PROMETHEE, são consideradas seis funções de preferência prédefinidas que cobrem a maioria das aplicações. As funções de preferência podem ser determinadas através da natureza do critério e/ou do ponto de vista dos tomadores de decisão 
[4]. Para este caso, a função utilizada em todos os critérios foi a Usual.

\section{RESULTADO}

De acordo com a estrutura proposta, o desempenho local de cada critério é utilizado para calcular a pontuação agregada final, que por sua vez defini o desempenho global das empresas de navegação estudadas. A Figura 4 apresenta o desempenho global obtido por meio da metodologia de agregação PROMETHEE II.

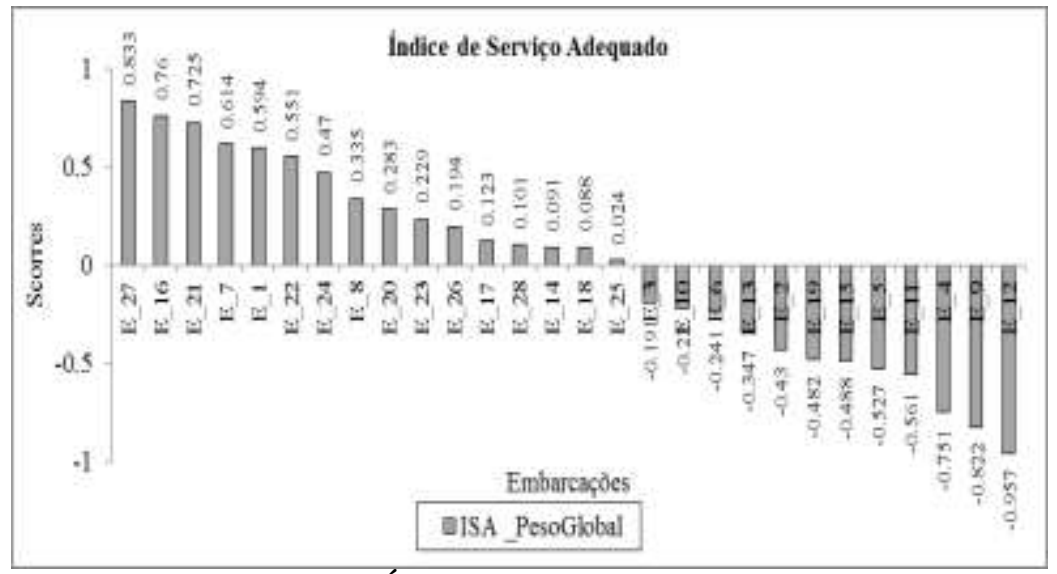

Figura 4: Índice de serviço adequado

Os pesos dos critérios são referentes ao peso global. Neste caso, a maior importância é dada ao aspecto segurança, seguido da alimentação, higiene, conforto, modicidade e atendimento. A seção 6.1 apresenta os resultados do desempenho global para os outros pesos (máximo e mínimo).

Na Figura 5 observa-se que, a embarcação E_27 tem o maior ISA $(0,833)$. Este resultado indica que a embarcação E_27 apresenta o melhor serviço adequando, ou seja, possui os maiores desempenhos locais nos critérios utilizados, exceto no critério modicidade (Vide Tabela 1). Já a embarcação E_12 tem o menor ISA (-0.957), apresentando os menores desempenhos locais nos critérios e, consequentemente, o pior serviço adequando.

\subsection{ANÁLISE de SENSIBILIDAdE}

A análise de sensibilidade é o estudo da estabilidade de critérios e alternativas na variação dos valores dos parâmetros. As comparações dos resultados finais obtidos por meio de diferentes pesos é uma forma de analisar a sensibilidade [12]. Desta forma, a Figura 5 apresenta os resultados do ISA para os pesos alternativos (máximo e mínimo).

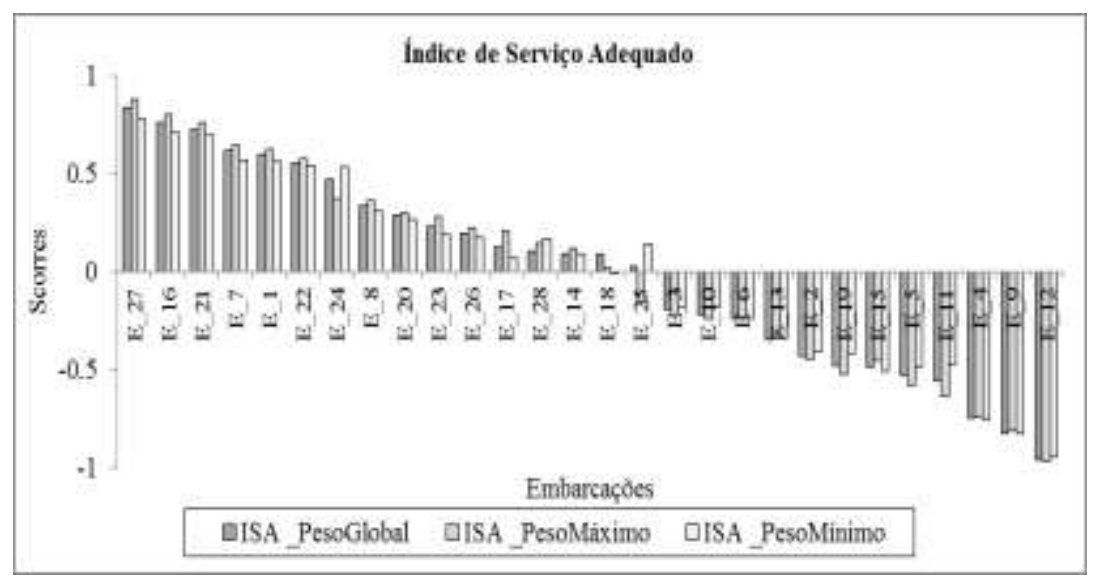

Figura 5: Análise de Sensibilidade/Robustez do ISA 
$\mathrm{Na}$ análise comparativa do ISA para os diferentes pesos observa-se que, a classificação das embarcações ficou muito similar, não havendo alterações dos resultados para um intervalo considerável de variações. Assim conclui-se que, o comportamento da classificação é robusto, pois as alternativas são pouco sensíveis a mudanças de preferências dos tomadores de decisão.

No caso aqui apresentado, observa-se que a embarcação E_27 tem os maiores valores para todos os ISA's, isso significa que esta é a alternativa que apresenta o melhor desempenho global e é a melhor solução de compromisso para atender corretamente os três "julgamentos".

\section{CONSIDERAÇÕES FINAIS}

A proposição de um índice global que avalia o desempenho das empresas de navegação mistas da Amazônia mostra-se válido no sentido de identificar referências para as menos eficientes. Para o caso aqui apresentado, verificou-se que a embarcação E_27 é a mais eficiente e pode ser usada com referência (benchmarks) para que as embarcações consideradas ineficientes melhorem seus desempenhos.

O resultado da aplicação da metodologia apresentada pode proporcionar uma melhoria do transporte fluvial de passageiros na Amazônia. A manutenção do indicador proposto oferece um diagnóstico de onde deve ser melhorado, influência no planejamento das políticas públicas, na regulamentação, qualificação e especialização do setor, além de fornecer alinhamento conceitual aos usuários.

Quanto à contribuição deste trabalho, espera-se que, por meio da utilização do ISA, os tomadores de decisão e usuários possam tomar decisões mais precisas, tais como: onde e quando melhorar o transporte fluvial de passageiros?

Como proposta para futuras pesquisas, sugere-se o desenvolvimento de um indicador que agregue o transporte de passageiros e carga uma vez que, as embarcações mistas são responsáveis por transportar os dois tipos de carga em uma mesma viajem. Assim, o indicador refletiria melhor as condições do sistema de transportes fluvial misto.

\section{AGRADECIMENTOS}

Este artigo é dedicado em memoria da Prof. ${ }^{\text {a }}$ Márcia Helena Veleda Moita, pela excelente pesquisadora e grande amiga. Os autores também agradecem a FAPEAM Fundação de Amparo à Pesquisa do Estado do Amazonas, a UFAM - Universidade Federal do Amazonas e o Instituto de Pesquisa em Transportes - INTRA pelo suporte aos membros envolvidos no desenvolvimento do artigo. 


\section{REFERÊNCIAS BIBLIOGRÁFICAS}

[1] ANTAQ. Resolução $\mathrm{N}^{\circ}$ 912, de 23 de novembro de 2007. Norma para Outorga de Autorização para Prestação de Serviço de Transporte de Passageiros e de Serviço de Transporte Misto na Navegação Interior de Percurso Longitudinal Interestadual Internacional. Brasília: Agência Nacional de Transportes Aquaviários - ANTAQ (2007).

[2] Brans, J.P. Vincke, P; Mareschal, B. How to select and how rank projects: the PROMETHEE method. European Journal of Operational Research, v. 24, p. 228-238, (1986).

[3] Brans, J.P. e Mareschal, B. Promethee-Gaia, une Methogologie de d'Aide à la Décision em Présence de Critères Multiples. Éditions Ellipses, Bruxelles, (2002).

[4] Brans, J.P. e Mareschal, B. Multiple Criteria Decision Analysis - State of the Art Surveys. Springer's Internacional Series. PROMETHEE METHODS Chapter 5, (2005).

[5] Couto, M. A. F. Proposição de indicadores de desempenho aplicado ao transporte de passageiros na região amazônica, Dissertação apresentada ao Programa de Pósgraduação em Engenharia de Produção Manaus/AM, (2008).

[6] Couto, M. A. F.; Moita, M. H. V.; Machado, V.W.; Kuwahara, N. Modelo não paramétrico aplicado à análise de eficiência do Transporte Aquaviário de Passageiros na Região Amazônica. In: XXIII ANPET - Congresso de pesquisa e Ensino em Transportes, Vitória, (2009).

[7] Duarte, R. C. D. S.; Kuwahara, N.; Alencar, L. A. Perspectiva Ergonômica para Embarcações do Estado do Amazonas. In: XXIII Congresso de Pesquisa e Ensino em Transportes XXIII ANPET, Vitória, (2009).

[8] Frota, C. D. Gestão da Qualidade Aplicada às Empresas Prestadoras do Serviço de Transporte Hidroviário de Passageiros na Amazônia Ocidental: Uma Proposta Prática. Tese (doutorado) - UFRJ/ COPPE/ Programa de Engenharia de Transportes, (2008).

[9] Kuwahara, N. Relatório Parcial do Projeto de Pesquisa intitulado Pesquisa e Desenvolvimento de Projetos de Embarcações Regionais na Amazônia. Projeto apoiado pelo fundo CTAQUAVIÁRIO / Edital MCT/CNPq/CT-Aquaviário no 08/2009 Transporte Aquaviário e Construção Naval. Número do processo no CNPq: 557131/2009-0, Manaus, (2011).

[10] Mareschal, B. e Brans J.P. Geometrical representations for MCDA. The GAIA Module. European Journal of Operational Research, 34:69-77, (1988).

[11] Merege. F. Indicadores da Navegação Hidroviária Mista na Região Amazônica. In: 7 SOBENA - Seminário de Transporte e Desenvolvimento Hidroviário Interior, Porto Alegre/RG. 7 SOBENA, (2011).

[12] Rigo, N., Hekkenberg R., Ndiaye A. B., Hadhazi D., Simongati G. e Hargitai C. Performance Assessment for Intermodal Chains. EJTIR, 7, no. 4, pp. 283-300, (2007).

[13] Saaty, T. L. Decision Making - The Analytic Hierarchy and Network Processes (AHP/ANP). Journal of Systems Science and Systems Engineering/ Vol. 13, No. 1, (2004). 